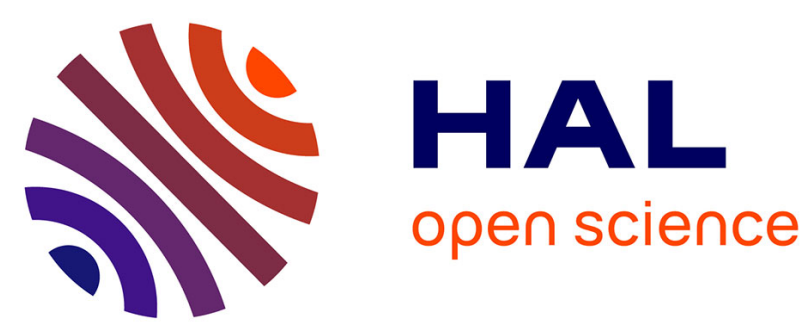

\title{
UTILISATION DE LA SPECTROSCOPIE DE PERTES D'ÉNERGIE D'ÉLECTRONS À L'IDENTIFICATION DE DÉPÔTS INTRA-RÉNAUX
}

M. Mignon-Conte, F. Carentz, J. Pourrat, J. Conte, Yolande Kihn, Jean Sévely

\section{To cite this version:}

M. Mignon-Conte, F. Carentz, J. Pourrat, J. Conte, Yolande Kihn, et al.. UTILISATION DE LA SPECTROSCOPIE DE PERTES D'ÉNERGIE D'ÉLECTRONS À L'IDENTIFICATION DE DÉPÔTS INTRA-RÉNAUX. Journal de Physique Colloques, 1984, 45 (C2), pp.C2-603-C2-606. 10.1051/jphyscol:19842141 . jpa-00223812

HAL Id: jpa-00223812 https://hal.science/jpa-00223812

Submitted on 1 Jan 1984

HAL is a multi-disciplinary open access archive for the deposit and dissemination of scientific research documents, whether they are published or not. The documents may come from teaching and research institutions in France or abroad, or from public or private research centers.
L'archive ouverte pluridisciplinaire HAL, est destinée au dépôt et à la diffusion de documents scientifiques de niveau recherche, publiés ou non, émanant des établissements d'enseignement et de recherche français ou étrangers, des laboratoires publics ou privés. 


\title{
UTILISATION DE LA SPECTROSCOPIE DE PERTES D'ÉNERGIE D’ÉLECTRONS A L'IDENTIFICATION DE DÉPÔTS INTRA-RÉNAUX
}

\author{
M. Mignon-Conte, F. Carentz, J. Pourrat, J.J. Conte, Y. Kihn ${ }^{*}$ et J. Seve1y \\ Laboratoire d'Imminopathologie Rénale et d'Immu-phamacologie, Service de \\ Néphrologie et d'Hémodialyse, C.H.U. Purpan, 31059 Toulouse, France \\ * Laboratoire d'optique Electronique du C.N.R.S., B.P. 4347, \\ 31055 Touzouse, France
}

Rësumé : La technique de microanalyse par spectroscopie de pertes d'énergie d'électrons est utilisẻe pour l'identification chimique de divers types de dépots intrarénaux. Elle a permis la détection de dépôts de béryllium, de silicium, d'iode, de fer et d'argent.

Abstract : Microanalysis by EELS has been used for the elemental identification of urinal deposits identification observed in fine pathological kidney sections. The technique has allowed the detection of béryllium, silicium, iodine, iron and silver deposits.

La technique de microanalyse par spectroscopie de pertes d'énergie d'électrons est utilisée pour l'identification chimique de dépots minéraux intra-rénaux. Cette méthode a permis jusqu'à ce jour de mettre en évidence, au cours de tableaux cliniques des éléments dont la responsabilité peut être discutée dans la recherche de l'origine de certaines maladies rénales.

Cette méthode s'applique sur des fragments de biopsie rénale humaine pratiquée à l'aiguille. Le tissu rénal est fixé au glutaraldehyde, cette opération est suivie d'une post-fixation osmique selon les techniques habituelles. L'Epon est le matériel d'inclusion. Les coupes sont non colorées ou colorées par l'acétate d'uranyle et le citrate de plomb. Leur épaisseur est comprise entre 190 et $240 \mathrm{~nm}$. ETles sont examinëes en microscopie électronique à haute tension (1 MV) (3). L'aire analysée étant définie par un diaphragme de sêlection de $10 \mu \mathrm{m}$ et correspondant à une surface de $100 \mathrm{~nm}$ de diamētre. Un repérage des dépôts intra-rênaux qui se présentent, soit sous la forme de sphères micro-cristallines, soit d'images de type lysosomial, est fajt sur les miorographies obtenues en microscopie électronique conventionnelle avant T'application de l'analyse par spectroscopie de pertes d'énergie $(4,5)$. Ainsi ont pu être analysées des particules dont les dimensions sont d'environ $30 \mathrm{~nm}$.

RESULTATS

L'observation privilêgiêe de deux malades présentant une glomérulonéphrite rapidement progressive au cours d'une silicose pulmonaire a donné lieu à l'identification de dēpôts de silicium. Les dépôts se trouvent au niveau des glomérules : dans les capsules de Bowman (Fig $1 A$ et $B$ ), les espaces urinaires, les axes mésangiaux.

Du béryllium a été trouvé dans une néphropatie apparemment primitive. Dans le parenchyme rénal, sa distribution est assez diffuse : basales tubulaires, tissu intertubulaire, capsules de Bowman, cellules épithéliales glomérulaires (Fig2 A et B) et axes mésangiaux.

Des dépôts d'argent sont retrouvés en quantité abondante, chez un malade longtemps traité par une thērapeutique à base de ce produit, mais sevré depuis de Tongues années, alors qu'apparait une insuffisance rénale. L'argent est situë dans les mitochondries tubulaires, (Fig $3 \mathrm{~A}$ et $\mathrm{B}$ ). 
De l'jode et du fer : s'associent dans les tubes rénaux d'un sujet recevant un traitement antiarythmique : le chlorhydrate d'amiodarone présentant par ailleurs une hëmochromatose. Ces dépôts sont découverts lors de 1 'examen de la biopsie rénale effectuee en raison d'une diminution de la fonction rénale. (La figure $4 \mathrm{~A}$ et B illustre d'une part la localisation des particules, d'autre part l'analyse chimique.)

On doit souligner qu'il n'a jamais été retrouvé aucun de ces dépots dans les reins normaux pris comme témoins.

Le rôle pathogène de ces dēpôts minêraux doit être bien entendu plus largement démontré par des méthodes plus directes : épidémiologiques ou expérimentales en particulier. Ces résultats sont déjà prometteurs et l'intervention de certains d'entre eux, le silicium, par exemple, comme point de départ de néphropathies à médiation immunologique reste posée.

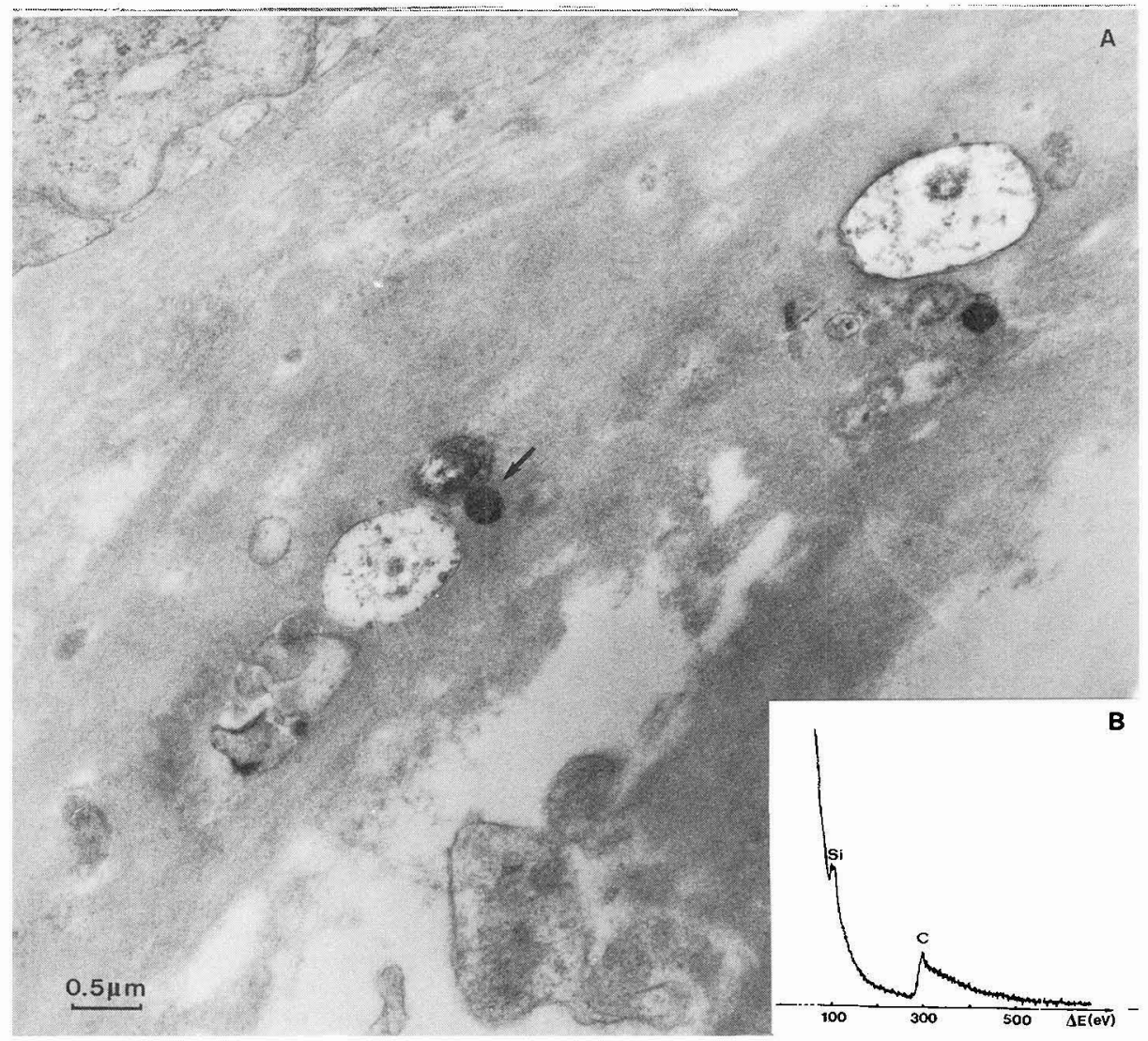

Fig. 1 : A - Dans la capsule de Bowman d'un glomérule, images sphêriques microcristallines contenant du silicium.

B - Spectre des pertes d'énergie obtenu à 1 MV sur 1 'amas sphérique fenêtre d'énergie $80-650 \mathrm{eV}$ - mise en évidence du silicium et du carbone. 

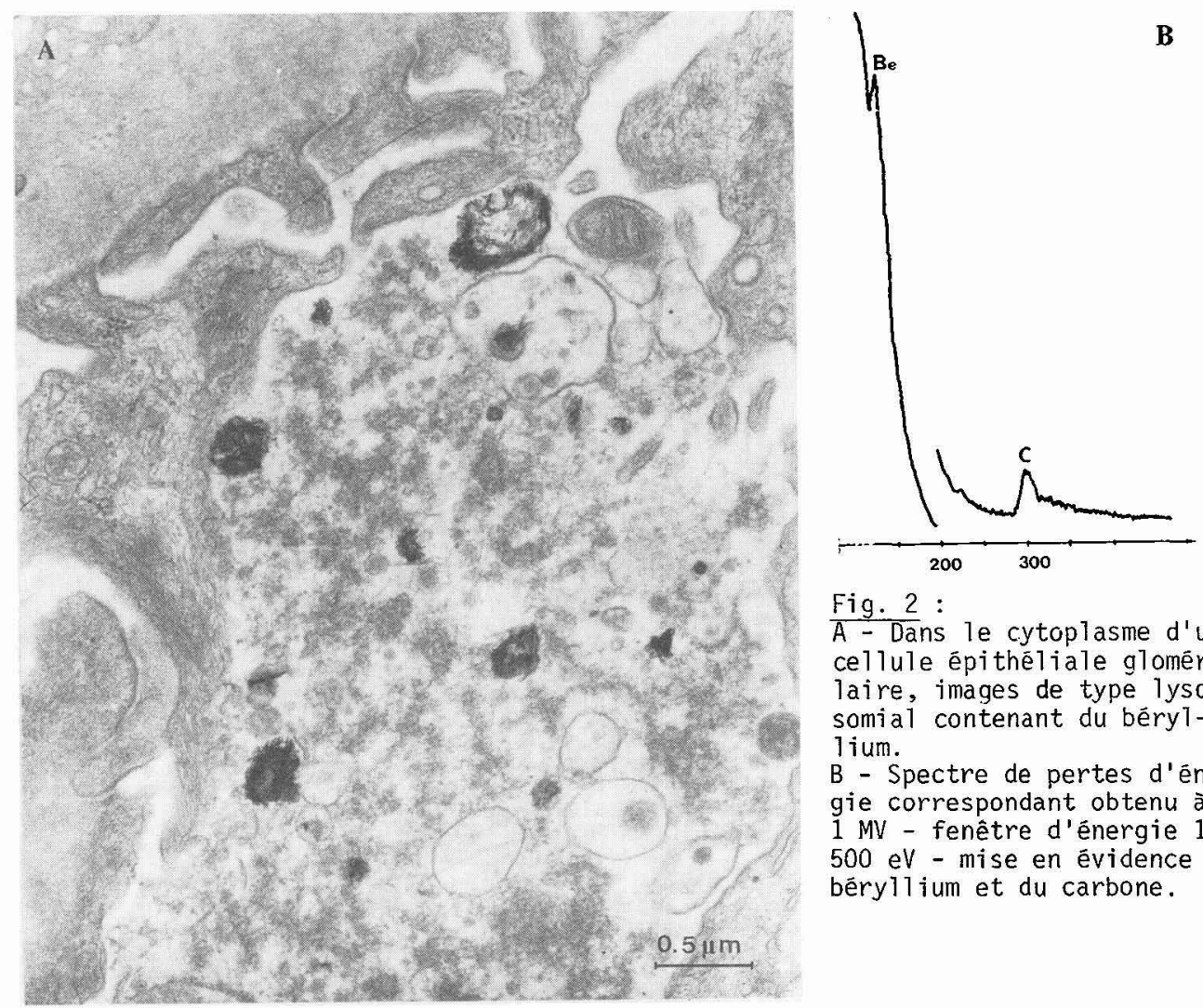

Fig. 2 :

A-Dans le cytoplasme d'une cellule épithéliale glomérulaire, images de type 1ysosomial contenant du béry Tlium.

B - Spectre de pertes d'ênergie correspondant obtenu à 1 MV - fenêtre d'énergie 100 $500 \mathrm{eV}$ - mise en évidence du béryllium et du carbone.
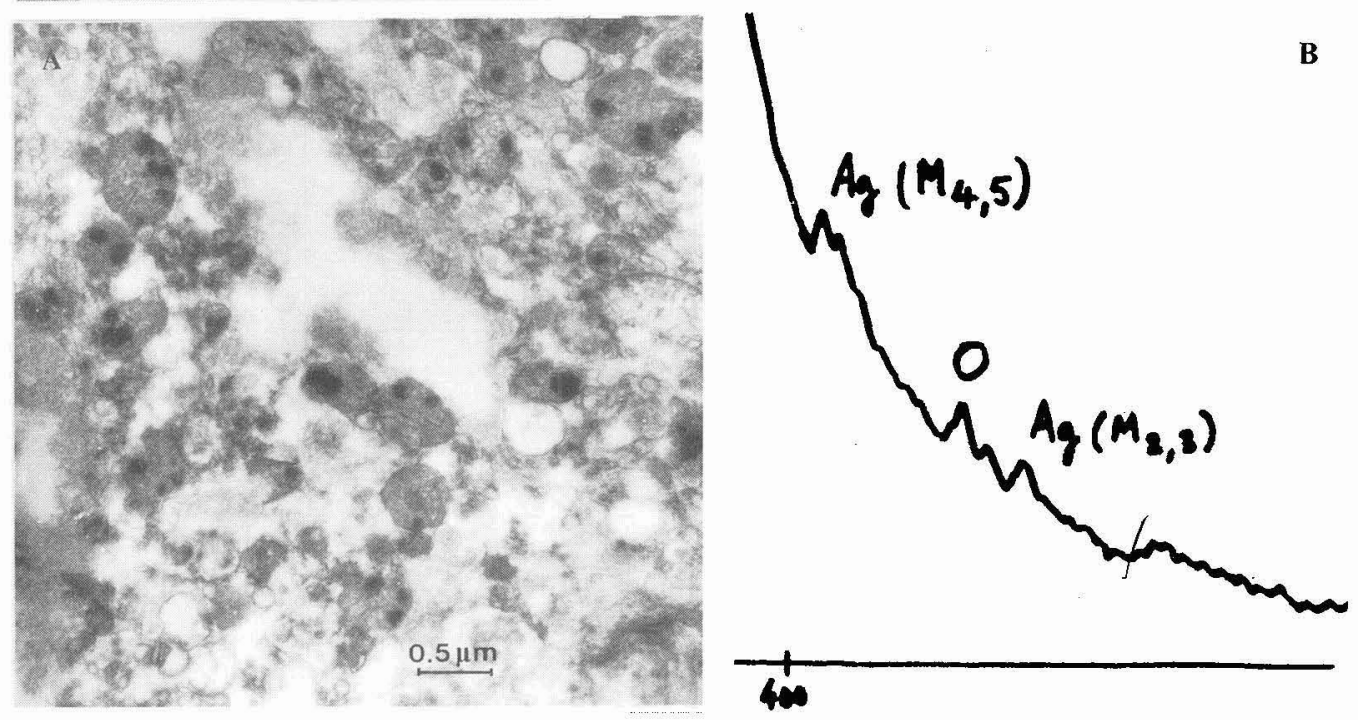

Fig. 3 : A - Localisation intramitochondriale de grains d'argent dans les tubes rênaux.

B - Spectre de pertes d'énergie associē - fenêtre d'énergie 400 $800 \mathrm{eV}$ - mise en évidence de l'argent et de l'oxygène. 


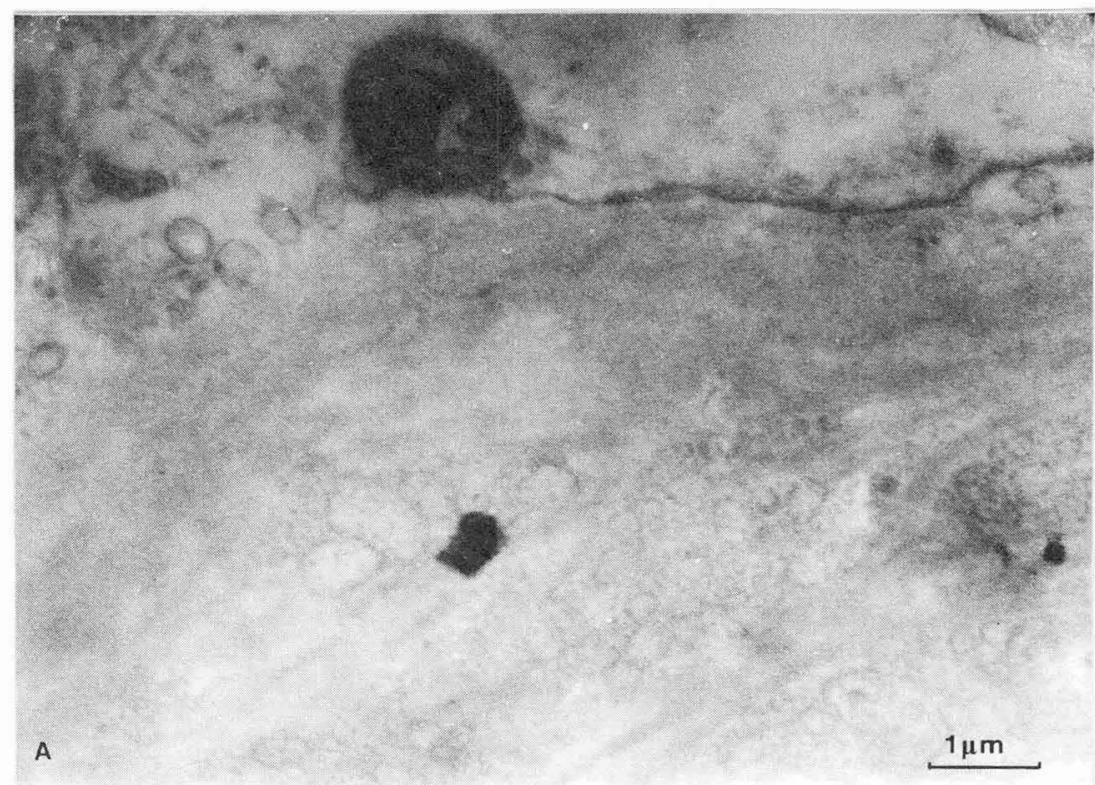

B

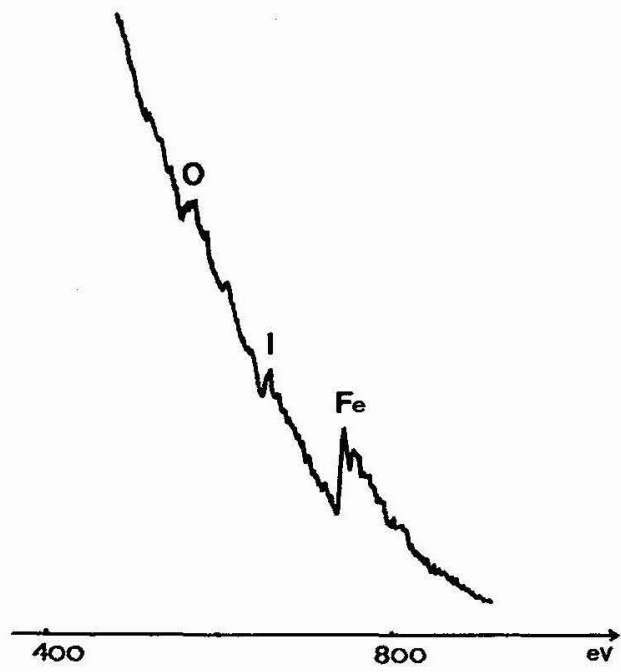

Fig. 4 :

A-[ocalisation intratubulaire d'un dépôt microcristallin.

$B$ - Spectre de pertes d'énergie associé fenêtre d'énergie $400-900 \mathrm{eV}$ - mise en évidence d'oxygène, d'iode et de fer.

\section{REFERENCES}

(1) J.B. BERRY, P. GALLE, P. HENOC and G. LAGRUE, Path. Biol., 25 (1977) 37.

(2) The Lancet, 1978, JuTy 1, 22.

(3) G. ZANCHI, Thèse Science, Université Paul Sabatier, Toulouse, France, Juillet 1978.

(4) J. SEVELY, Le Vide, 191 (1978) 69.

(5) R.F. EGERTON, Proc. 10 th International Congress on Electron Microscopy, Hamburg 1 (1982) 151. 